\title{
A Motion Model of a Remotely Operated Underwater Vehicle: CFD Simulation
}

\author{
D. Thai Nguyen ${ }^{1}$, V. Horák ${ }^{2 *}$, L. The Nguyen ${ }^{1}$, \\ D. Van Nguyen ${ }^{1}$ and L. Do Duc ${ }^{1}$ \\ ${ }^{1}$ Le Quy Don Technical University, Hanoi, Vietnam \\ ${ }^{2}$ University of Defence, Brno, Czech Republic
}

\begin{abstract}
The manuscript was received on 8 March 2021 and was accepted after revision for publication as research paper on 17 September 2021.
\end{abstract}

\begin{abstract}
:
The article is directly related to the authors' previous study of the remotely operated underwater vehicle's (ROV) motion, where the mathematical model of the ROV motion is derived for laminar flow of water. This solution of the ROV motion has been improved by using the three-dimensional computational fluid dynamics (CFD) simulation that allows simulating the turbulent flow, which is mainly generated by propellers. Both methods were coupled to obtain more accurate values of hydrodynamic damping coefficients for the improved determination of the ROV motion velocity. Results of the solution are discussed and some of them are compared with the experiment.
\end{abstract}

\section{Keywords:}

CFD simulation, hydrodynamics, remotely operated vehicle, turbulent water flow

\section{Introduction}

Remotely operated underwater vehicles (ROVs) are underwater robots that are widely used in science, military, industries and entertainment. The topic of the article is directly related to the authors' previous study of the ROV motion [1]. Here, the ROV motion equations are complemented by hydrodynamic parameters and forces acting upon the underwater ROV. In addition, the matrices of hydrodynamic damping coefficients and external forces acting upon the ROV are considered in this study.

This mathematical model of the ROV motion is derived under the assumption of laminar flow of water. The mentioned solution of the ROV motion can be improved by using the CFD simulation that allows simulating the turbulent flow. Turbulence is mainly generated by propellers. Turbulence in the water flow may influence the mo-

\footnotetext{
* Corresponding author: Department of Mechanical Engineering, University of Defence, Brno, Kounicova 156/65, CZ-662 10 Brno, Czech Republic. Phone: +420 973442 616, E-mail: vladimir.horak@unob.cz.ORCID 0000-0003-1654-7441.
} 
tion of all types of underwater vehicles, including ROV - mainly their velocity. Higher values of the ROV velocity cause turbulent flow behind the main body and between the body and propellers.

CFD simulation makes it possible to understand how and to specify where turbulence is generated. One of the goals of the presented CFD simulation is to determine the axial and cross-flow hydrodynamic coefficients. The outcomes of the ROV motion CFD simulation are coupled with the Fortran ROV motion model [1] to obtain more accurate results.

\section{Three-Dimensional CFD Simulation of ROV Motion}

\subsection{Virtual Prototype Model of ROV}

The side view of the underwater ROV structure consisting of the main body, two lights, two thrusters and two balance flotation blocks is shown in Fig. 1. The main body has the shape of a cylindrical vessel with hemispherical ends. The characteristic dimensions are length $L$ and diameter $D$. The underwater ROV structure dimensions are given in Tab. 1.

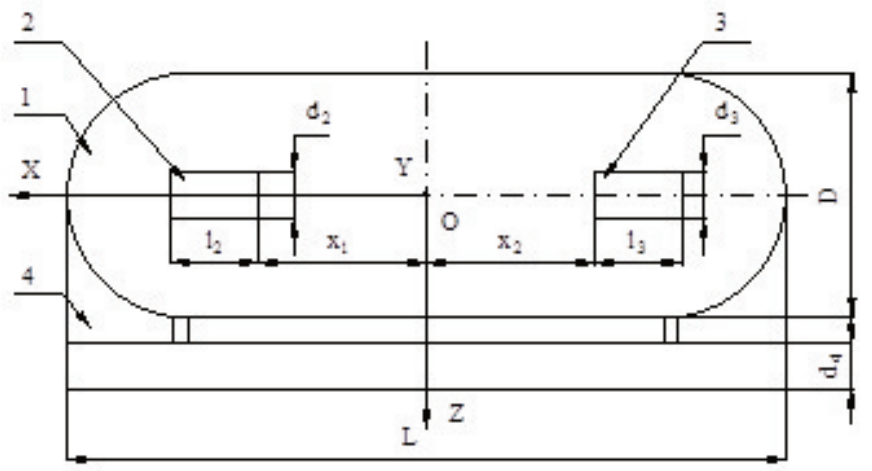

Fig. 1 Underwater ROV structure: 1 - main body, 2 - light, 3 - thruster, 4 - balance flotation block

Tab. 1 ROV dimensions

\begin{tabular}{|l|c|c|c|}
\hline \multicolumn{1}{|c|}{ Input parameter } & Symbol & Unit & Value \\
\hline Length of the ROV & $L$ & $\mathrm{~m}$ & 0.80 \\
\hline Diameter of the ROV main body & $D$ & $\mathrm{~m}$ & 0.25 \\
\hline Length of the light & $l_{2}$ & $\mathrm{~m}$ & 0.11 \\
\hline Diameter of the light & $d_{2}$ & $\mathrm{~m}$ & 0.06 \\
\hline Length of the thruster & $l_{3}$ & $\mathrm{~m}$ & 0.102 \\
\hline Diameter of the light & $d_{3}$ & $\mathrm{~m}$ & 0.097 \\
\hline Length of the balance flotation block & $l_{4}$ & $\mathrm{~m}$ & 0.80 \\
\hline Diameter of the balance flotation block & $d_{4}$ & $\mathrm{~m}$ & 0.086 \\
\hline Radius of the two hemispheres & $D / 2$ & $\mathrm{~m}$ & 0.40 \\
\hline Distance from end of light to origin $O$ & $x_{1}$ & $\mathrm{~m}$ & 0.15 \\
\hline Distance from top of thruster to origin $O$ & $x_{2}$ & $\mathrm{~m}$ & 0.10 \\
\hline
\end{tabular}


The 3D virtual prototype model of the complex-shaped underwater ROV is built by using the SolidWorks computer-aided engineering software. The concept of the 3D ROV model is shown in Fig. 2. The thruster of the ROV can be installed to rotate counter clockwise.
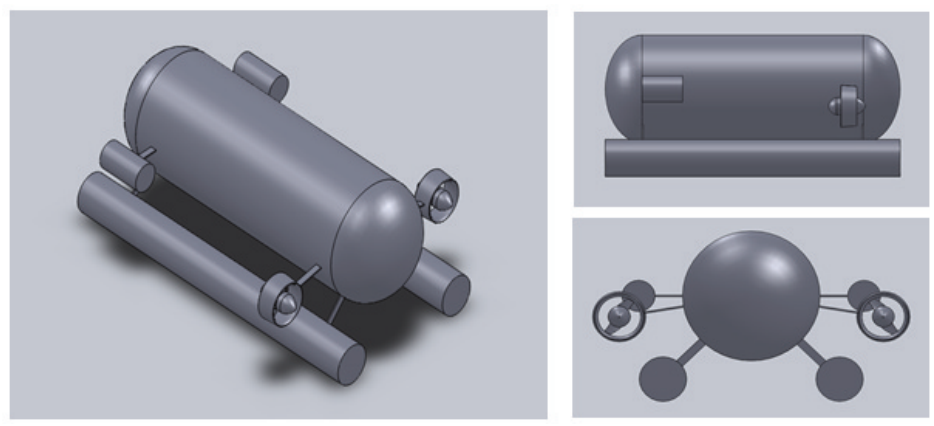

Fig. 2 Concept of 3D ROV model

\subsection{Meshing Process}

After importing the 3D underwater ROV model from SolidWorks program, the computational mesh was generated by using the ANSYS, which is a general-purpose meshing tool that leverages the libraries from the more advanced ANSYS meshing modules [2]. The computational mesh is generated by using the collection of edges, vertices and faces that define the shape and size of the model.

The structured mesh, in regions of interest near the ROV surface, is more detailed than in the others to capture the water flow around the ROV. As the underwater ROV virtual prototype has the port-starboard plane symmetry, it is possible to simulate only half of the model and recalculate the results for the full ROV model after that.

The main domain around the ROV model was created by the tetrahedral mesh with the minimum size of elements form $1.5 \mathrm{~mm}$ to $3 \mathrm{~mm}$. This minimum size of elements is capable to detect the effect of the complexed structure areas of the underwater ROV.

The entire length of the semi-cylindrical domain area is $5.4 \mathrm{~m}$ and the radius is $1.8 \mathrm{~m}$. Domain dimensions were used in accordance with [3]. The mesh elements are growing in a direction perpendicular to the surface of the ROV body into the fluid. The mesh was smoothed to ensure a continuous transition between elements. This is important for calculating the transition from laminar to turbulent flow. The mesh of the main domain around the ROV model is shown in Fig. 3.

In order to increase the accuracy of the simulation, the mesh elements of the area which is near the underwater ROV surface and around complex shaped thrusters (see Fig. 4) need to be generated manually with the help of body sizing and face sizing functions.

Parameters of the body sizing and face sizing functions are as follows: The dimension of the body elements is around $2 \mathrm{~mm}$ and the minimum body element size is $0.1 \mathrm{~mm}$. The dimension of the face elements is about $1 \mathrm{~mm}$ and the minimum face element size is also $0.1 \mathrm{~mm}$. The detailed mesh around the thruster body and for the propeller is shown in Fig. 4. 


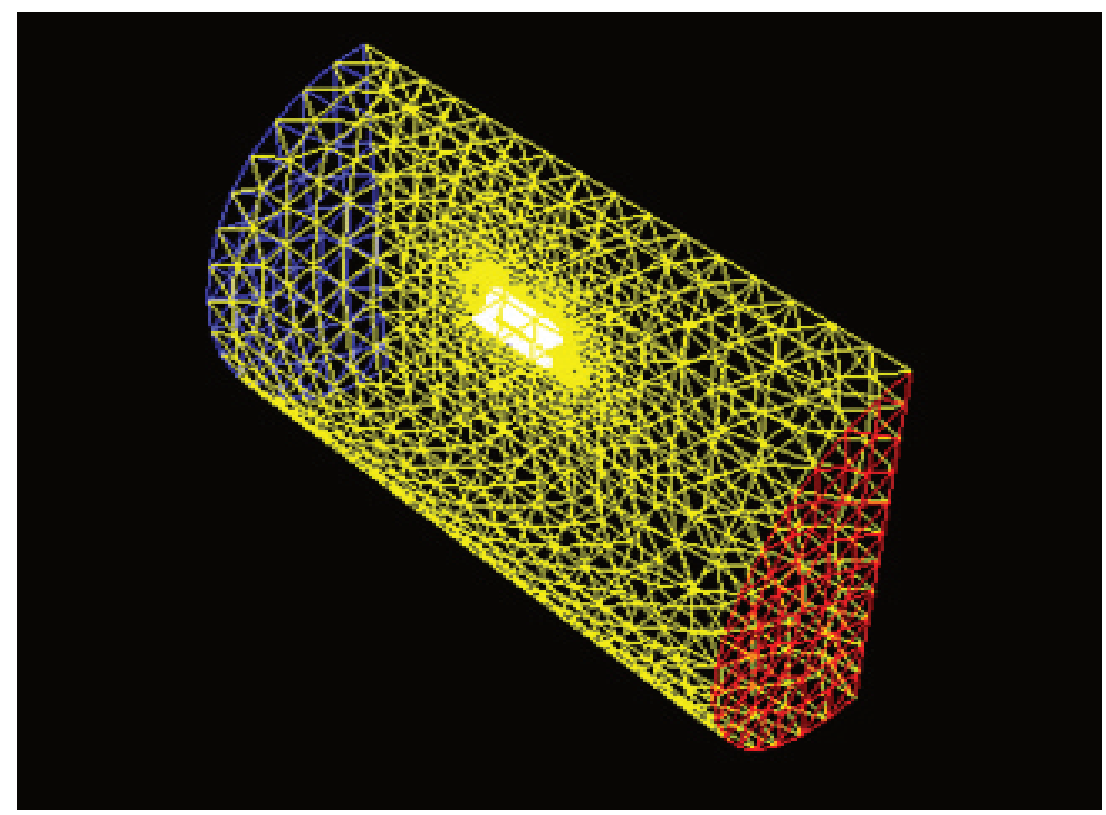

Fig. 3 Mesh of main domain around underwater ROV model

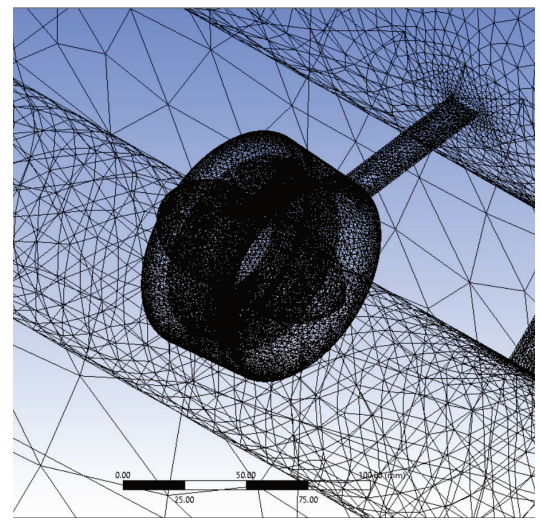

a)

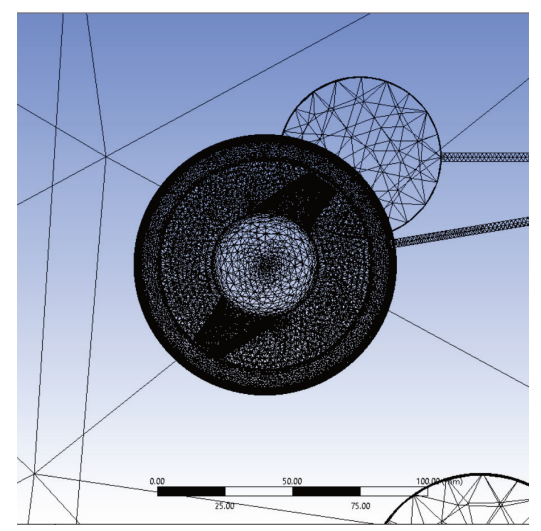

b)

Fig. 4 Detailed mesh: a) around thruster, b) for propeller

Meshing for the complete area of the numerical domain, which covers one half of the 3D underwater ROV model, includes above 1.66 million of elements. The view of the complete structural computational mesh is shown in Fig. 5.

\subsection{Boundary Conditions}

The boundary operating conditions of simulation and the domain inlet and outlet parameters are as follows: 
- the ambient fluid is water of the constant density of $998.2 \mathrm{~kg} / \mathrm{m}^{3}$, the dynamic viscosity is $0.001003 \mathrm{~Pa} \cdot \mathrm{s}$, and the temperature of $20^{\circ} \mathrm{C}$,

- the water flow is without heat transfer,

- the overpressure of fluid is $10000 \mathrm{~Pa}$,

- the free stream velocity in $X$ - and $Z$-axis directions is changing from zero until a final value according to the time-dependent external procedure,

- the free stream velocity in the $Y$-axis direction is $0 \mathrm{~m} / \mathrm{s}$,

- the low free stream turbulent intensity of flow is $5 \%$, which corresponds to the flow characteristics of free stream in the experimental channel,

- the used turbulence model is $R N G k-\varepsilon$, which is the most widely used and validated turbulence model in CFD.

The calculation run setup is given by:

- the time step which equals $0.0002 \mathrm{~s}$. This time step was selected based on the experience with the calculation so that the solution provides sufficiently accurate results within a reasonable computation time,

- the number of time steps is 100000 .

The ROV motion velocity is here as the input parameter to the CFD simulation program through the velocity components in $X$ - and $Z$-axis directions. This velocity varies during the time of the ROV motion. The coordinate system is defined in Fig. 1.

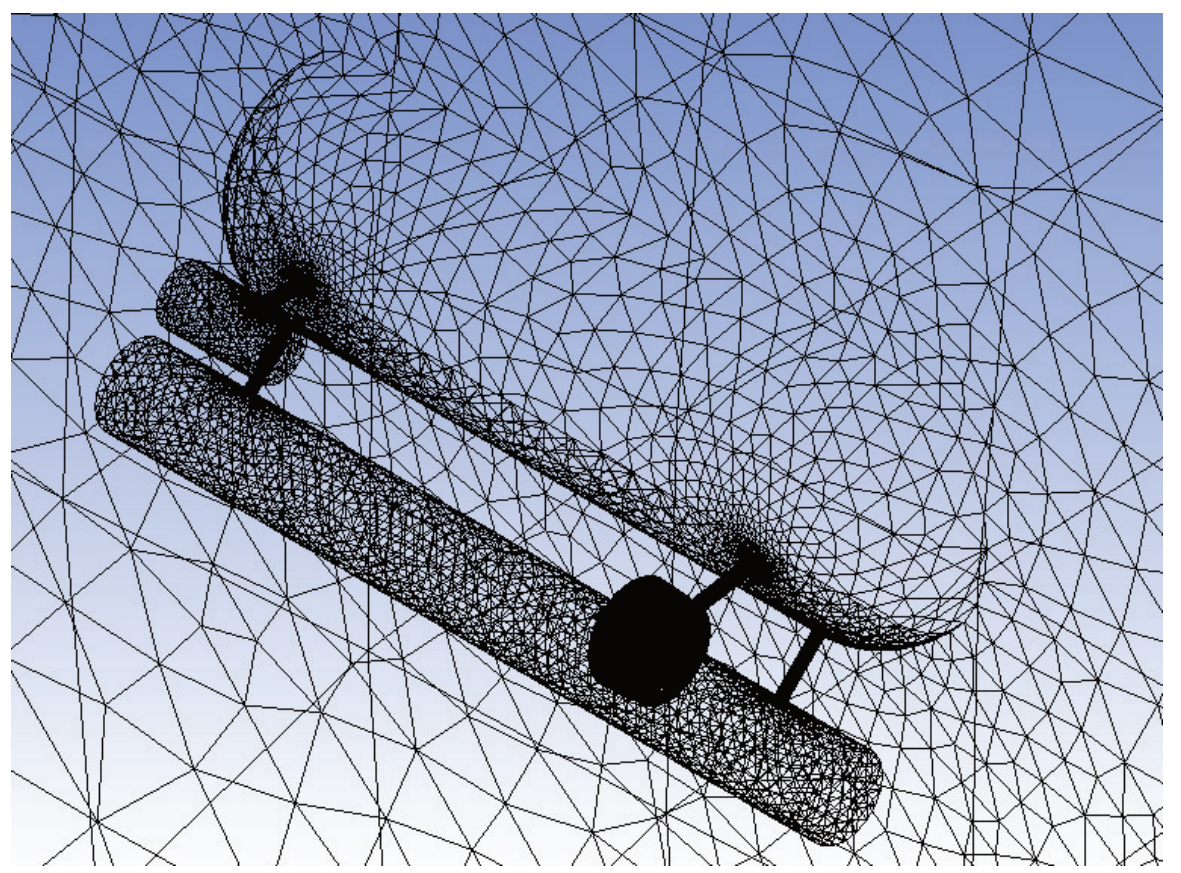

Fig. 5 Computational mesh of the $3 D$ underwater ROV model

\subsection{Results of CFD Simulation}

The ANSYS Fluent CFD simulation provides illustrative results enabling a thorough study of the fluid flow around the underwater vehicle. The velocity and the pressure distributions in the investigated flow are needed to determine the effects of external 
hydrodynamic forces acting upon the underwater ROV body. The following figures show some examples of various modes of the velocity and the pressure distributions at a time of 20 seconds after the beginning of the ROV motion. It corresponds with the final free stream velocity of $1.62 \mathrm{~m} / \mathrm{s}$. The particular propeller speed is set up so that the thruster force is $5,10,15$, and $20 \mathrm{~N}$, respectively. This thruster force range corresponding to the propeller speeds from 300 to 420 rounds per minute was determined by experiments.

Streamlines of the fluid flow in the plane passing through the thruster axes are shown in Fig. 6. Here, one can see that the flow velocity attains its highest value at the exit from the thrusters.

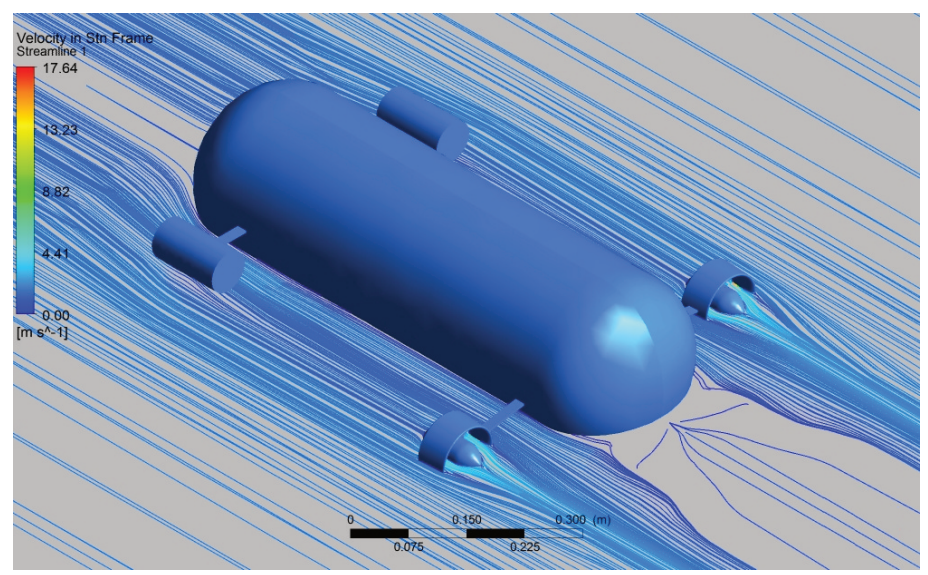

Fig. 6 Streamlines in the plane passing through thruster axes

The corresponding velocity distribution of the flow around the underwater ROV in the $X O Y$ plane passing through thruster axes is shown in Fig. 7. In this figure, one can clearly observe various areas of flow: the maximum velocity at the exit from the thrusters, the backward fluid flow behind the underwater ROV main body, and indicate the magnitude and orientation of the free stream velocity that corresponds to the ROV motion velocity.

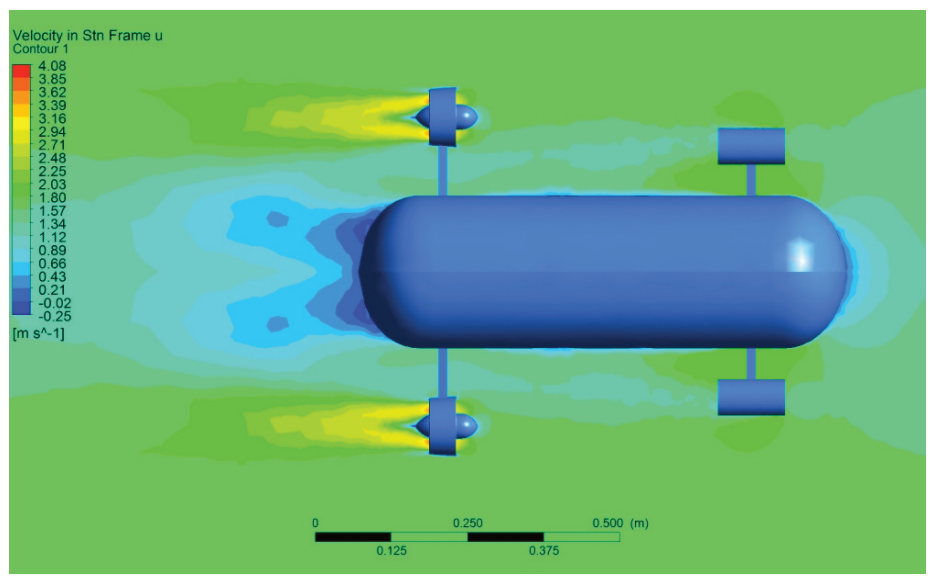

Fig. 7 Velocity distribution in XOY plane 
The velocity distribution in the cross direction, i.e. on the plane $Y O Z$ is shown in Fig. 8. Here, one can see the upward flow increasing buoyancy between the balance flotation blocks under the underwater ROV main body and indicate the magnitude and orientation of the free stream velocity.

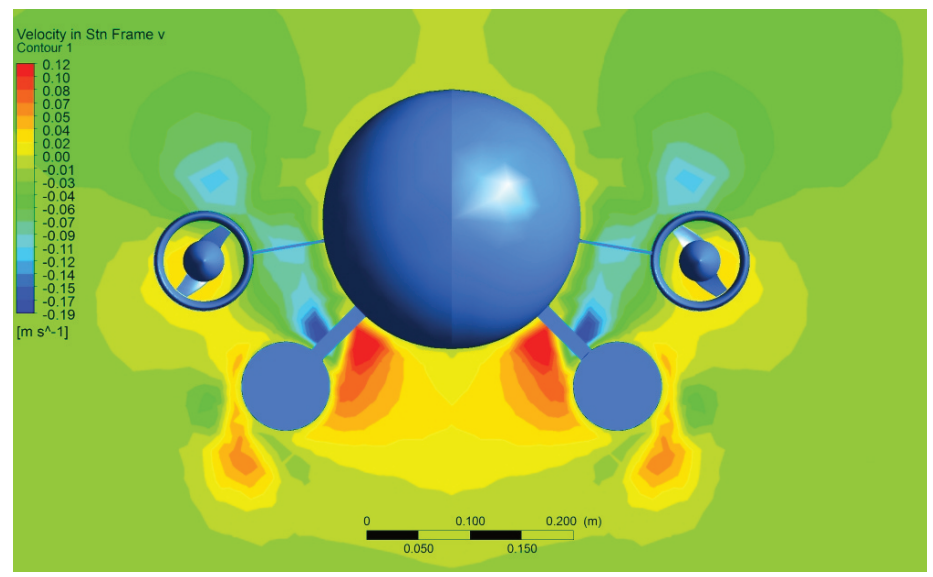

Fig. 8 Velocity distribution in plane $Y O Z$

The velocity distributions shown in Figs 6, 7 and 8 allow a detailed study of the water flow through thrusters and flow around surfaces of the individual parts of the ROV, when the latter moves under water.

Examples of the overpressure distribution around the underwater ROV are shown in Figs 9 and 10. As expected, the highest pressure is on the leading side of the ROV. The thrusters also substantially affect the pressure distribution.

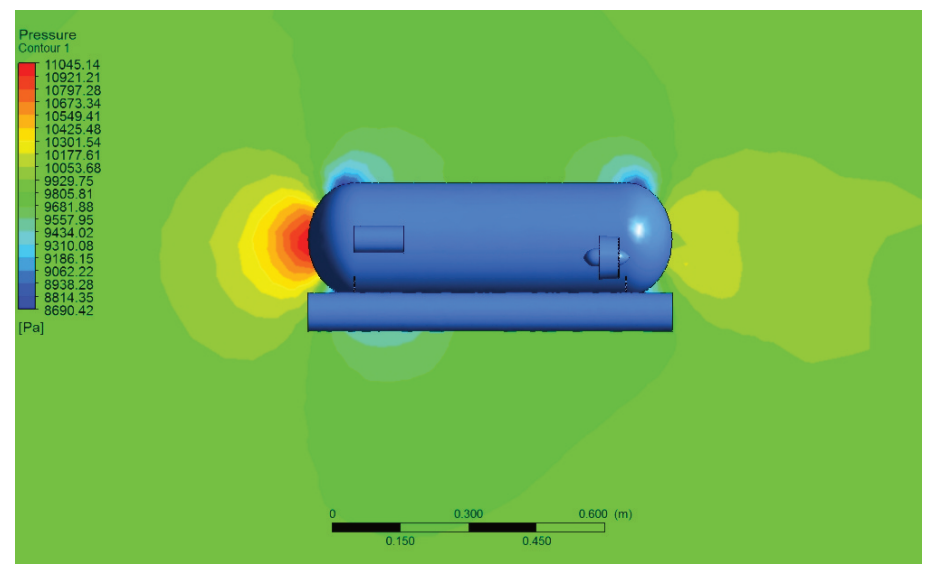

Fig. 9 Pressure distribution on plane XOY

The hydrodynamic pressure forces acting upon the underwater ROV are then obtained by the integration of the pressure over the whole ROV surface. Components of these forces in the axial and cross flow directions determine the axial and cross-flow hydrodynamic coefficients respectively used for calculating the quadratic damping coefficients in the Fortran ROV motion model given in the authors' previous study [1]. 


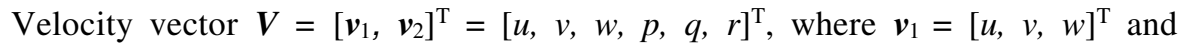
$\boldsymbol{v}_{2}=[p, q, r]^{\mathrm{T}}$ are the translational and rotational velocities of the ROV in the bodyfixed coordinate system $(O, X, Y, Z)$. Here, $u, v, w$ are the translational velocity components and $p, q, r$ are the rotational velocity components of the ROV motion in the body-fixed coordinate system $O, X, Y, Z$.

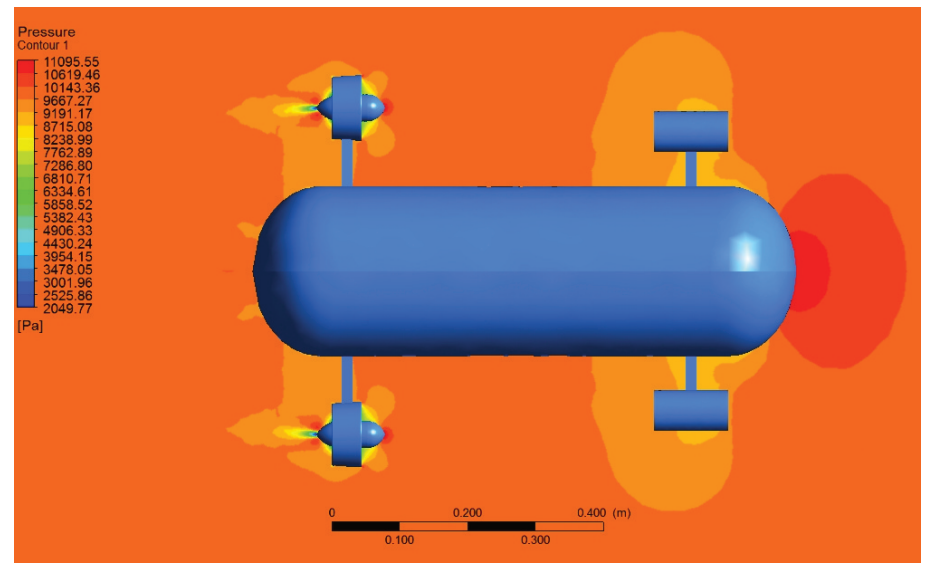

Fig. 10 Pressure distribution on plane XOZ

\section{Coupled Model of Underwater ROV Motion}

The improved couple model simulating the underwater ROV motion is the combination of two sub-models:

- the sub-model of the underwater ROV motion using the matrices of hydrodynamic damping coefficients and external forces acting upon the ROV. The solution obtained by the Runge-Kutta method is achieved by using the Fortran programming language [1],

- the sub-model simulating the flow velocity and pressure distribution by using the above ANSYS Fluent CFD simulation.

The schema of the coupling model is shown in Fig. 11. The Outside Program (OP) that calculates the ROV's velocity vector $\boldsymbol{V}=(u, v, w, p, q, r)^{\mathrm{T}}$ is realized at the same steps as the CFD simulation. Data exchange between OP and CFD program is performed by means of the Memory Management System (MMS). The coupling model uses the following routines: the Createinput.F which writes the computed values to MMS and the Readmms.F that is giving the data back to the CFD sub-model. The CFD simulation program (ANSYS Fluent) is connected with the external code (Fortran) automatically and simultaneously $[4,5]$.

The coupling process of both sub-models can be described by the following steps:

1. at the beginning, the Createinput.F function is called for the first time in order to create the data of ROV velocity $\boldsymbol{V}$ and the water velocity $\boldsymbol{V}_{\text {water, }}$, and to save input parameters such as length, diameter, mass, moments of inertia, water density, etc. to the MMS for outside programming,

2. next, the OP is called at the point of the Start of Time Step. The input values are provided from Createinput.F function, if it is the first time step or by the previous step, 
3. once OP is called, OP is running to determine the ROV velocity vector $\boldsymbol{V}$. This process is shown by the black dashed line on the right side of Fig. 11,

4. after the simulating process, OP writes back the ROV data $V$ to MMS. This process is shown by the red dashed line on the right side of Fig. 11,

5. while the CFD coefficient loops are performed, the function Readmms.F is called several times to obtain ROV motion data from the MMS. These data are the input parameters for the CFD simulation,

6. once CFD simulations are completed, the program returns the result of the CFD calculation, which is the water flow velocity $\boldsymbol{V}_{\text {water }}$,

7. the calculated $\boldsymbol{V}_{\text {water }}$ from the step 6 is written to the MMS using the Createinput.F function. This value of $\boldsymbol{V}_{\text {water }}$ serves as the input data for OP at the next step. This process is shown by the third red line on the left side of Fig. 11.

When the step 7 is finished, the calculation process comes back to the step 2 to create a loop calculation.

The evolution of the ROV velocity vector component along the $O_{0} X_{0}$ axis is shown in Fig. 12 for the above-mentioned coupled model in comparison with the noncoupled model for the case of propulsive force $20 \mathrm{~N}$. The non-coupled model of the ROV motion is calculated by using only the Fortran program [1] without the CFD simulation.

Based on the results shown in Fig. 12, the velocity along $O_{0} X_{0}$ axis of the coupled model is slightly lower than the one of the non-coupled model. The comparison of the final velocities of the ROV motion with the experiment for the distance between the thrusters $0.505 \mathrm{~m}$ and for the case of propulsive force $20 \mathrm{~N}$ of each thruster is given in Tab. 2.

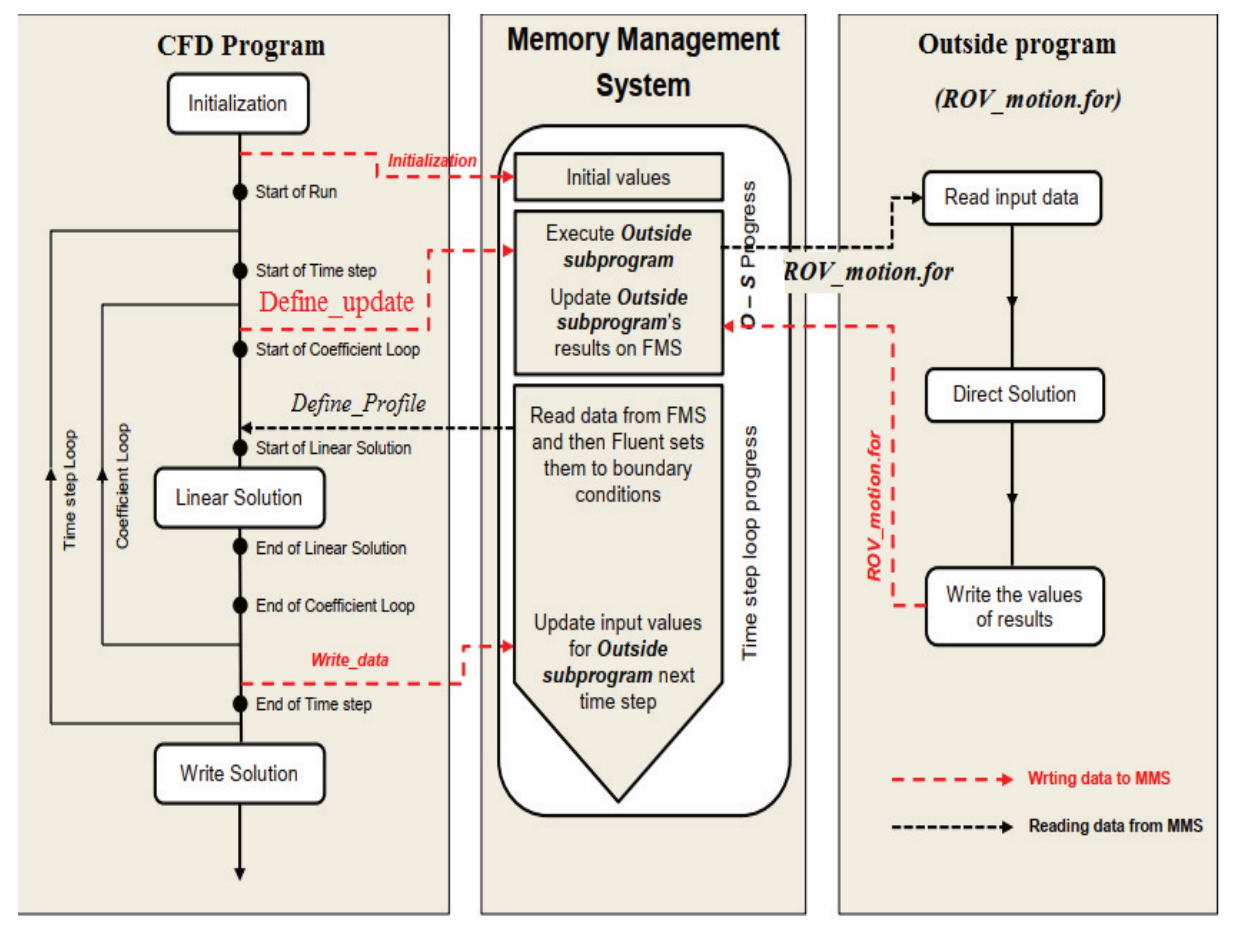

Fig. 11 Scheme of data management between sub-models 


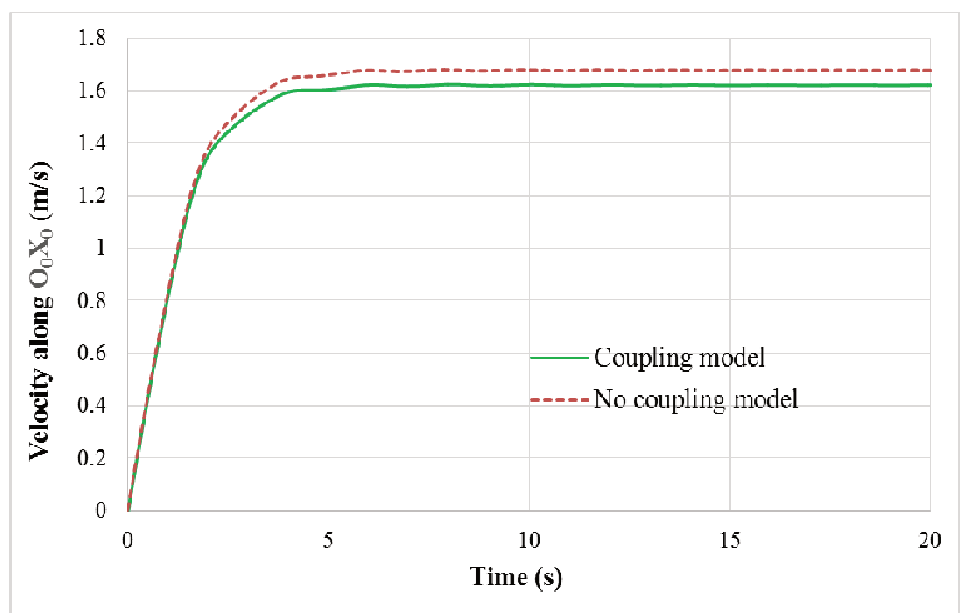

Fig. 12 Velocity comparison of coupled and non-coupled model

Tab. 2 Comparison of ROV final velocities for coupled and non-coupled model

\begin{tabular}{|c|c|c|c|c|}
\hline \multicolumn{5}{|c|}{ ROV motion final velocity along $\boldsymbol{O}_{\mathbf{0}} \boldsymbol{X}_{\mathbf{0}}[\mathrm{m} / \mathrm{s}]$} \\
\hline Experiment & Coupled model & Difference [\%] & $\begin{array}{c}\text { Non-coupled } \\
\text { model }\end{array}$ & Difference [\%] \\
\hline 1.56 & 1.62 & 3.85 & 1.65 & 5.77 \\
\hline
\end{tabular}

The velocity of the underwater ROV is measured by a high-speed camera. The experiment is described in detail in the previous study [1], where the velocity along $O_{0} X_{0}$ axis was measured, other velocity components were neglected for the purpose of this study. The comparison in Tab. 2 shows that the presented coupled model of the ROV motion is more realistic than the non-coupled model. That is because, the CFD flow simulation allows to include multiple influences into the solution, such as the turbulence of water flow, the velocity of the surrounding water stream [6], etc. This allows to draw a conclusion that the coupled model provides more accurate results than the non-coupled model.

\section{Conclusion}

The described ANSYS Fluent CFD simulation of the flow around the underwater ROV allows a detailed description of the water flow around its parts. The resulting velocity and pressure distributions in the investigated flow are needed to determine the axial and cross-flow hydrodynamic coefficients used for calculating the quadratic damping coefficients in the Fortran ROV motion model [1].

Although the requirements on the hardware and the computation time are substantially higher, the coupled procedure of both sub-models allows for a more precise determination of velocity components in comparison with the previous non-coupled model. The latter has been verified for the case of the final velocities of the ROV motion by experiment. 
The presented coupled procedure enables us to obtain deeper understanding of the underwater ROV motion for designing and controlling UAV.

\section{Acknowledgement}

Authors gratefully acknowledge the support from the fund of the Le Quy Don Technical University under grant number BKHCN-DTDLCN.45/20 and the institutional funding DZRO FVT 3 of the University of Defence.

\section{References}

[1] THAI NGUYEN, D., V. HORÁK, H. THU TRAN, L. THE NGUYEN and C. QUANG HOANG. A Motion Model for a Complex-Shaped Remotely Operated Underwater Vehicle. Advances in Military Technology, 2020, 15(2), pp. 343-353. DOI 10.3849/aimt.01403.

[2] ANSYS Fluent User's Guide, Release 15.0 [online]. November 2013 [viewed 2021-03-04]. Available from: http://www.pmt.usp.br/academic/martoran/ notasmodelosgrad/ANSYS\%20Fluent\%20Users\%20Guide.pdf

[3] BADAWY, A.M. and A.A. OMER. Dynamic Analysis of Remotely Operated Underwater Vehicle Model. International Journal of Engineering Science Invention, 2013, 2(7), pp. 5-16. ISSN 2319-6734.

[4] REINECKE, E.-A., S. KELM, W. JAHN, C. JÄKEL and H.-J. ALLELEIN. Simulation of the Efficiency of Hydrogen Recombiners as Safety Devices. International Journal of Hydrogen Energy, 2013, 38(19), pp. 8117-8124. DOI 10.1016/j.ijhydene.2012.09.093.

[5] THU TRAN, H., T. DUC NGUYEN, D. THAI NGUYEN and H. NGOC DUONG. Super Cavity Model with the Coupling Reaction of Slender Body Motion and Water Flow. Vietnam Journal of Mechanics, 2018, 40(1), pp. 1-13. DOI 10.15625/0866-7136/8488.

[6] THE NGUYEN, L., T. NGUYEN VAN, H. THU TRAN and T. DUC NGUYEN. Influence of the Water Velocity on a Remotely Operated Vehicle Motion. In: Proceedings of the $5^{\text {th }}$ International Conference on Engineering Mechanics and Automation (ICEMA 5). Hanoi: University of Engineering and Technology, 2020, pp. 59-66. ISBN 978-604-9955-18-1. 